\title{
EL DESARROLLO DE HUMILDAD CULTURAL MEDIANTE EL APRENDIZAJE SERVICIO CRÍTICO
}

\author{
DEVELOPING CULTURAL HUMILITY THROUGH \\ CRITICAL SERVICE LEARNING
}

\author{
KAREN LUCAS BREDA* \\ KIM Groot $^{* *}$ \\ ANTOINETTE TOWLE ${ }^{* * *}$
}

\begin{abstract}
RESUMEN
Millones de personas en los EE. UU. experimentan marcadas desigualdades en los servicios de salud que reciben. La segregación racial urbana y la estratificación social separan a los grupos sociales y dificulta la comunicación. Muchos profesionales de la salud de los Estados Unidos mantienen estereotipos negativos hacia la gente marginada de las zonas urbanas. Es necesaria la creación de vehículos para el entendimiento y el respeto entre los profesionales de salud y sus pacientes. La humildad cultural utiliza la autoreflexión, la autocrítica, la apertura y la trascendencia para abordar las desigualdades de poder. El "aprendizaje servicio crítico" (Critical Service Learning) es un tipo de "aprendizaje servicio" que adopta los principios de la teoría crítica para la formación de consorcios enfocados a fomentar la responsabilidad y justicia social. Este artículo describe el Proyecto Horizonte (Project Horizon), una colaboración innovadora de promoción de la salud y defensa social con base en una universidad que utiliza el aprendizaje servicio crítico en una comunidad urbana para fomentar la humildad cultural. Los resultados del Proyecto Horizonte demuestran que este consorcio es un vehículo eficaz para el desarrollo y la mejora de la humildad cultural entre los profesionales de la salud que participan en el esfuerzo. Implicaciones: Los proyectos de promoción social y de servicios de salud como el Proyecto Horizonte pueden ser reproducidos en otros lugares para aumentar la capacidad de desarrollo de enfermeras y otros profesionales de la salud con orientación democrática y poseedores de una mayor sensibilidad a la humildad cultural.
\end{abstract}

Palabras clave: Humildad cultural, aprendizaje servicio crítico, personal de salud, disparidades en atención de salud, consorcios de salud.

\begin{abstract}
Marked inequities or disparities in health care and health outcomes exist for millions in the United States of America. Urban apartheid and increasing social stratification separate social groups making it difficult for them to understand each other. Many U.S. health care professionals, including nurses, hold negative stereotypes toward the urban poor and disenfranchised. Creating vehicles for understanding and respect between health care professionals and the social groups they care for is needed. Cultural humility uses self-reflection, selfcritique, openness and transcendence to address power inequities between providers and clients. "Critical service-learning" is a type of "service-learning" that adopts the tenets of critical theory and pedagogy in engaged partnerships to foster social responsibility and a justice-oriented framework. The purpose of this article is to showcase Project Horizon, an innovative university-based health and social advocacy partnership that uses critical service learning with the urban community to foster cultural humility. Project Horizon outcomes show

\footnotetext{
* Enfermera. University of Hartford, West Hartford, Connecticut. College of Education, Nursing and Health Professions, Institute for Translational Research, Center for Health, Care and Well-Being, Project Horizon. Email: breda@hartford.edu

${ }^{* *}$ Enfermera. University of Hartford. West Hartford, CT 06117 USA. Email: groot@hartford.edu

${ }^{* * *}$ Enfermera. Southern Connecticut State University. New Haven, CT 06515 USA. Email: Towlea1@southernct.edu
} 
that this long-term engaged partnership is an effective vehicle for developing and enhancing cultural humility among health care professionals participating in the endeavor. Implications: Replication of social and health advocacy projects such as the one conducted through Project Horizon can be made in other settings to increase the capacity for democratically oriented nurses and other health care professionals who have an enhanced sense of cultural humility.

Key words: Cultural humility, critical service learning, health personnel, healthcare disparities, health consortia.

Fecha recepción: 12/09/12 Fecha aceptación: 27/05/13

\section{INTRODUCCIÓN}

Es innegable que muchas personas en los Estados Unidos de Norteamérica (EE.UU.) sufren de condiciones de desigualdad en las áreas de economía y salud. Numerosos reportes federales de EE.UU. dan a conocer este problema social constante e implacable (1-4). Desigualdades dramáticas como las que se detallan en los informes apenas mencionados influyen negativamente en la capacidad de las personas para mantenerse saludables, conectadas socialmente y económicamente seguras. Las intervenciones nacionales a "nivel macro" en los EE.UU. pueden beneficiarse mucho en caso de realizarse importantes cambios estructurales diseñados para revertir las desigualdades sociales y permitir una composición social más equitativa. Las propuestas progresistas de reformas sociales y de salud se han visto frustradas en las últimas décadas por políticas económicas neoliberales que restringen la intervención pública y presionan por una creciente privatización, desregulación e individualización. Las políticas económicas neoliberales favorecen principalmente a los más ricos y con frecuencia tienen consecuencias devastadoras para los pobres (5).

Entre las soluciones locales (nivel micro) a las condiciones sociales adversas en los EE.UU. se incluyen las iniciativas de voluntariado organizadas por grupos constituidos sin fines de lucro. La intención de hacer el bien y ver por los demás de una manera caritativa esta muy generalizada en los EE.UU. Aunque bien intencionadas, la mayoría de las iniciativas a nivel micro carecen de una clara conexión con los grandes problemas estructurales a nivel macro. Estas iniciativas a nivel micro son a menudo fragmentadas y no cuentan con una conexión racional entre sí y con el todo. La comprensión de los problemas estructurales de la economía política y de la conexión entre soluciones a niveles macro y micro es un elemento clave en la creación de iniciativas durables y significativas que generen cambios positivos que permitan mejorar la democracia (6).

La estratificación social y la ideología de clase a menudo hace que los grupos sociales se mantengan separados y atemorizados entre sí. En los Estados Unidos, las separaciones urbano-suburbano y urbano-rural se han generalizado con las poblaciones pobres de color concentradas en barrios urbanos abandonados situados lejos de los sitios donde vive la gente adinerada que son principalmente los suburbios con población mayoritariamente blanca (7). Esta segregación racial -apartheid urbano- (separación geográfica en la raza y la clase social) no es sólo una separación física de los grupos de población, sino también una separación ideológica, racial, étnica y de clase. Para hacer frente a estas desigualdades se necesitan iniciativas a nivel macro nacionales y cambios importantes a nivel estructural, tanto en la política económica como en los sistemas de servicios 
de salud y sociales. Al mismo tiempo, se necesitan iniciativas a nivel micro en los barrios y las comunidades locales para dar a diversos grupos la oportunidad de participar en la acción, para entender la dimensión humana de los problemas, y para la creación de soluciones viables e innovaciones. A fin de cuentas, la combinación de iniciativas de niveles macro y micro, en conjunto con una población bien informada, es lo que puede ayudar a una sociedad a crear y sostener el cambio.

Desafortunadamente, es más fácil decirlo que hacerlo. Un buen número de estadounidenses en la población general carecen de una comprensión de las desigualdades sociales y políticas, y por lo general no participan en esfuerzos diseñados para promover la justicia social o reducir las disparidades. Su percepción de las cuestiones de clase, raza y género sociales está determinada por la ideología dominante, que incluye los valores dominantes en los Estados Unidos de la competencia, la independencia y el individualismo. Los profesionales de la salud de los Estados Unidos, inclusive las enfermeras, al igual que muchos estadounidenses de la población en general, tienden a tener un conocimiento limitado de las cuestiones sociales, políticas y estructurales generales que generan y estimulan las desigualdades de raza, clase social y de género. A menudo se culpa a las víctimas por sus circunstancias, tales como a la gente pobre, por ser pobre o los que abusan de las drogas por su falta de control. Esta falta de conciencia social y política crea una fractura entre los profesionales sanitarios y las poblaciones a las que sirven. En consecuencia, los profesionistas de la salud de Estados Unidos frecuentemente son incapaces de comprender $\mathrm{u}$ ofrecer atención verdaderamente democrática a personas de diversas etnias, razas, niveles socioeconómicos, nacionalidades o religiones.

La implementación de programas de educación y curriculares especialmente diseñados para las enfermeras y otros profesionales de la salud que van más allá de la enseñanza de la competencia cultural enfocándose en fomentar la humildad cultural es un vehículo para el desarrollo de profesionales de la salud con orientación democrática. El propósito de este artículo es describir el Proyecto Horizonte, una colaboración innovadora de servicios de salud y defensa social con base en una universidad que utiliza el método de aprendizaje servicio con perspectiva crítica dentro de comunidades urbanas para fomentar la humildad cultural (8).

\section{Humildad cultural versus competencia cultural}

El concepto de la humildad cultural está siendo utilizado cada vez más en la literatura de los EE.UU. relacionada con el servicio social, la salud y la educación. La humildad cultural se ha definido como "el proceso continuo de autorreflexión y autocrítica que aborda abiertamente las desigualdades de poder que existen entre los proveedores de servicios y sus clientes" (9). La humildad cultural va más allá de la noción de "competencia cultural", que es la idea de alcanzar el conocimiento experto sobre un grupo cultural.

La competencia cultural es un concepto bien conocido en la práctica de la enfermería en los EE.UU. y precedió el concepto de humildad cultural. La competencia cultural se centra en la comprensión de los atributos culturales de un grupo en particular, y en la adquisición de conocimientos acerca de estos atributos. Recientemente, el concepto de competencia cultural ha sido criticado por algunos analistas, debido a su falta de atención a las dimensiones sociales, políticas y de clase existentes en la sociedad. Por ejemplo, la competencia cultural no requiere que uno cuestione o intente cambiar las desigualdades de poder que existen entre el profesionista y su cliente. Enseñarles a los profesionales de la salud a que aprendan a conocer una lista de los atributos culturales de un grupo (competencia cultural) sin que entiendan las dinámicas de poder y las desigualdades que 
subyacen a su posición en la sociedad puede, sin intentarlo, servir para reforzar las relaciones de poder arraigadas que existen entre los profesionales sanitarios y sus clientes.

A diferencia del concepto de competencia cultural, la humildad cultural atiende a las dimensiones políticas, sociales y de clase que existen en la sociedad. La humildad cultural es más una forma de ser al estar con otros que tratar de conocer una lista de los atributos culturales de un grupo particular. La humildad cultural requiere que el profesional de la salud esté dispuesto a cambiar la forma en que aprende sobre otras personas e incluye las dimensiones de la autoconciencia, la apertura y la trascendencia (10).

El alcanzar un alto grado de humildad cultural permite a los profesionales desarrollar relaciones con la comunidad basadas en el respeto, la dignidad y la confianza. En su artículo sobre la humildad cultural entre las parteras de dos países (República Dominicana y los EE.UU.), Foster (11) señala la importancia de las colaboraciones comprometidas a largo plazo en contraste con las experiencias de inmersión episódicas. Foster postula que para que las conexiones entre profesional sanitario y sus clientes sean democráticas y para que el intercambio de ideas sea recíproco, es importante crear relaciones sustentables y de largo plazo. Es precisamente a través de este tipo de relación que puede desarrollarse la humildad cultural.

Cruess et al. (12) discuten la necesidad de que los profesionales sociales, de salud y educación desarrollen un contrato social con la sociedad. Un contrato social incluye un imperativo moral por parte del profesional a ser digno de confianza, altruista y a hacer lo que es correcto para la sociedad. Estos elementos se convierten en parte de la conciencia moral del profesional, la cual evoluciona con el tiempo. Además, mientras que los atributos tales como la atención y la compasión no pueden ser obligatorios o forzados, éstos pueden ser modelados por expertos y con- vertirse así en parte de las expectativas que los profesionales tienen de sus colegas y también de las expectativas que tiene la sociedad de dichos profesionales (12).

Los teóricos críticos sostienen que las posiciones de privilegio y poder están embebidas en la sociedad y que la discriminación, la parcialidad y los prejuicios a menudo permanecen ocultos a nuestra conciencia inmediata (6). La búsqueda de la humildad cultural es un primer paso que permite a los profesionales de salud la capacidad de comprender sus propias posiciones de poder sobre los clientes que interactúan con ellos y también el papel que desempeñan en la reproducción de estas relaciones, por ocultos que éstos se encuentren.

El proporcionar oportunidades para un diálogo abierto entre profesionistas de la salud y las personas que viven en las comunidades que los necesitan es esencial para ayudar a que dichos profesionistas se vuelvan sensibles a las necesidades de los demás y fomentar la creación de la humildad cultural y el desarrollo de practicantes con orientación democrática (11). El aprender a escuchar a las personas necesitadas para generar ideas relacionadas con sus padecimientos (en lugar de decirle a la gente lo que debe hacer de una manera paternalista) es una tarea difícil para los profesionistas de la salud. El hacer esto sin juzgar ni ver con lástima a la gente es aún más difícil. El solo reunir a profesionistas de la salud con personas de diferentes clases, grupos étnico y/o racial puede ayudar a todas las partes interesadas a reflexionar sobre los estereotipos embebidos y los prejuicios implícitos.

\section{¿Qué es el aprendizaje servicio?}

El aprendizaje servicio tradicional se ha utilizado durante varias décadas en los Estados Unidos, Canadá y Gran Bretaña, así como en otros países para poner a los estudiantes universitarios y a los profesionales en contacto con comunidades y poblaciones necesitadas. 
Bamber y Hankin (13) atribuyen al aprendizaje servicio el "renacimiento del interés en el papel de la virtud cívica, la responsabilidad y el servicio social en la educación superior". La conciencia y promoción de la salud en los EE.UU. pueden beneficiarse de estar vinculadas a las iniciativas de promoción de la salud y defensa social a través del aprendizaje servicio. El aprendizaje servicio puede ser pensado como una "pedagogía que dirige el aprendizaje del aula hacia la atención de las necesidades de las comunidades pertinentes (mediante el servicio social), en forma tal que la reciprocidad entre la institución y el socio comunitario es esencial en la formulación de propuestas, soluciones y estrategias para el cumplimiento de sus misiones organizacionales" (13).

Levesque-Bristol et al. (14) estudiaron la eficacia del aprendizaje servicio, centrándose en el entorno del aprendizaje y los resultados educativos de los estudiantes. Los autores encontraron que los participantes detectaban un ambiente más positivo cuando su percepción de autonomía, competencia e interrelación eran elevados. Los estudiantes que se percibían a sí mismos como poseedores de un cierto grado de autonomía y capacidad de toma de decisiones dentro de entornos de aprendizaje servicio, aumentaban su capacidad de resolver problemas, su acción cívica y su comprensión de la diversidad; los estudiantes que pensaban que tenían las habilidades para ayudar, tenían un mayor sentido de competencia. Los estudiantes que estuvieron directamente involucrados y conectados con las personas en un entorno aprendizaje servicio dieron una alta calificación a su nivel de interrelación personal. Esto aumenta la probabilidad de que los estudiantes perciban que pueden hacer realmente una diferencia. En el estudio de Levesque-Bristol et al. (14) todos los elementos juntos (autonomía, competencia y relación) causaron una mejora en la percepción de los estudiantes de la experiencia del aprendizaje servicio.

\section{¿Por qué aprendizaje servicio con perspectiva crítica?}

El aprendizaje servicio con perspectiva crítica o, más brevemente, aprendizaje servicio crítico (ASC) es un tipo especial de aprendizaje servicio que añade los componentes de la teoría y pedagogía críticas al enfoque tradicional del aprendizaje servicio. El ASC incluye un mayor aprendizaje experiencial (a través del servicio), la formación de consorcios y asociaciones comprometidas, está embebido dentro de un "marco orientado hacia la justicia" y tiene la ventaja de permitir que los estudiantes se "conviertan en agentes de igualdad social a través de un análisis crítico" (15). El uso del aprendizaje servicio crítico aumenta la eficacia del aprendizaje servicio tradicional pues estimula a que los participantes piensen más allá del ámbito local y así puedan examinar a un nivel más elevado los componentes macroestructurales que están detrás de la inequidad y así darse cuenta cómo es que cuestiones sociales, políticas y económicas sostienen y reproducen prejuicios de raza, clase y género. Por otra parte, el SAC sugiere que los propios participantes no sólo cuestionen el status quo sino que también busquen convertirse en divulgadores de cambio social (15).

\section{El Proyecto Horizonte como modelo de aprendizaje servicio}

Los consorcios de aprendizaje servicio crítico establecidos en las universidades pueden llegar a ser exitosos vehículos para aumentar la conciencia y la comprensión de las inequidades en salud y, últimamente, instrumentos promotores del cambio y democratización de la conducta de estudiantes y profesionales de salud, inclusive de las enfermeras. A continuación se describe en detalle el consorcio entre una universidad y las comunidades vecinas denominado Proyecto Horizonte, el cual es un programa de aprendizaje servicio crítico con base universitaria que propor- 
ciona un marco para la mejora de la salud y el conocimiento social a través de acciones y cambios basados en evidencias realizados entre poblaciones en riesgo.

El Proyecto Horizonte es una iniciativa de acercamiento a la comunidad y de aprendizaje servicio crítico establecida en el Centro para la Salud, Atención Sanitaria y el Bienestar del Instituto de Investigación Traslacional de la Facultad de Educación, Enfermería y Profesiones de la Salud de la Universidad de Hartford. Desde 1985, los profesores y los estudiantes de Licenciatura en Ciencias en Enfermería (BSN) de la Universidad de Hartford en West Hartford, Connecticut, EE.UU. han ofrecido atención médica gratuita y servicios de educación sanitaria en escuelas y agencias locales. En 1988, los servicios se ampliaron para incluir refugios locales para gente sin hogar, comedores comunitarios para gente necesitada y centros de vivienda transitoria.

Aunque el Proyecto Horizonte acoge a estudiantes de toda la universidad que desean colaborar en sus iniciativas, este artículo se centra sólo en los participantes que son alumnos del programa de enfermería que permite a las enfermeras registradas (RN) alcanzar el grado de Licenciatura en Enfermería (BSN) de la misma universidad. Estos estudiantes del programa de Licenciatura en Ciencias de la Enfermería de la Universidad de Hartford están ya registrados legalmente como enfermeras o enfermeros ante el Estado, poseen el Grado de Asociado en Enfermería (ADN) y están actualmente inscritos en cursos programados para que completen la licenciatura en enfermería (BSN). Para participar en el programa de BSN es requisito que los estudiantes del programa sean enfermeras o enfermeros registrados (RN) en el Estado de Connecticut, EE.UU.

Como parte de un curso de aprendizaje servicio comunitario de dos semestres relacionado con la preparación para la salud pública de la comunidad dentro del progra- ma de BSN, los estudiantes participan en el Proyecto Horizonte de cuatro a seis horas por semana como voluntarios en refugios para gente sin hogar, comedores comunitarios para gente necesitada, centros vecinales, centros de asistencia a desamparados y escuelas en barrios pobres. Debido a que los estudiantes del Proyecto Horizonte son enfermeras(os) profesionales, ellos pueden prestar servicios de enfermería de acuerdo con su licencia de RN y la Ley de Práctica de Enfermería del estado de Connecticut.

Para ayudar a describir las actividades universitarias y comunitarias del Proyecto Horizonte hemos dividido la trayectoria del Proyecto Horizonte en tres fases: La Fase 1 es la de orientación, construcción de relaciones y donde se aprende a reflexionar; la Fase 2 involucra la formación del consorcio y el desarrollo del proyecto, y la Fase 3 es la de implementación, evaluación y sostenibilidad.

\section{Proyecto Horizonte - Fase 1: Orientación, construcción de relaciones y reflexión del aprendizaje}

El objetivo principal del aprendizaje servicio crítico es fomentar la responsabilidad social y el desarrollo de un marco orientado hacia la justicia social entre los profesores y los estudiantes. Para lograr este objetivo, la pedagogía crítica y la teoría del aprendizaje de los adultos se utilizan en el Proyecto Horizonte para promover la práctica reflexiva e inspirar asociaciones de aprendizaje comprometidas. La pedagogía crítica y el aprendizaje experiencial han infundido el plan de estudios de enfermería en la Universidad de Hartford por más de veinticinco años. En consecuencia, las filosofías tanto de la unidad académica de enfermería y el Proyecto Horizonte se complementan y se informan mutuamente. Los programas académicos y de aprendizaje servicio comunitario de la Universidad de Hartford trabajan juntos para generar un compromiso general con la responsabilidad social y la práctica orientada a la justicia en- 
tre los estudiantes. Los cursos en el programa de Licenciatura en Ciencias en Enfermería están organizados para que se apoyen mutuamente. Cuando los estudiantes llegan a la etapa del practicum del aprendizaje servicio crítico del Proyecto Horizonte en el último año de estudios, están ya bien familiarizados con el aprendizaje comprometido y la pedagogía crítica.

Para preparar a los estudiantes para entrar en la experiencia del aprendizaje servicio en el Proyecto Horizonte, los profesores ofrecen cursos sobre la diversidad y la salud, sobre las disparidades sociales y de salud y sobre los determinantes sociales de la salud. Los estudiantes son sensibilizados a la existencia de los prejuicios y al racismo, así como también a los papeles del género, la etnicidad y la clase social y a la distribución desigual del poder.

Una orientación participativa para estudiantes que dura un día a la semana durante tres semanas se lleva a cabo antes de la entrada en la configuración del aprendizaje servicio comunitario. Durante esta orientación se crean equipos de estudiantes y profesores y un profesor presenta una serie de ejercicios de formación de equipos a los estudiantes. Entre las actividades de formación de equipos se incluyen un curso de desafío con cuerda donde cada estudiante sube 14 metros en el aire por cuerdas sostenidas por los otros estudiantes, una búsqueda de tesoros en la ciudad, visitas a museos de arte, exposiciones de fotografía y/o asistencia al teatro musical o dramático en vivo. Algunas de estas actividades se ofrecen en años alternos.

Los ejercicios de formación de equipos se eligen deliberadamente para ser diferentes, para alentar a los estudiantes a pensar "fuera de lo normal" y generar un sentido de la creatividad y descubrimiento y estimular el ingenio. El método está destinado a ayudar a los estudiantes y profesores a romper con el estilo educativo de la enfermería tradicional y alentarlos a buscar fuera de la disciplina ideas creativas para resolver problemas y para pensar en nuevas formas. Los ejercicios de formación de equipos también tienen como objetivo enseñar a los estudiantes a desarrollar, integrar y tejer su papel como proveedores de cuidado de la salud con los papeles de abogado, artista, activista, académico y ciudadano.

Un primer paso para ayudar a las enfermeras y otros profesionistas sanitarios a modificar su comportamiento con las poblaciones marginadas es aumentar su nivel de conocimiento y conciencia frente a las determinantes sociales de la salud y los aspectos sociales, históricos y político-económicos de la salud. Los profesores del Proyecto Horizonte entrenan a los estudiantes sobre estos temas. Además, animan a los estudiantes a sentirse más cómodos con la ambigüedad y que se abstengan de hacer juicios precipitados sobre personas y eventos.

El Proyecto Horizonte utiliza la pedagogía crítica como marco general para llevar a cabo sus principales resultados. Al hacer esto, se reestructuró el proceso educativo y formativo y se repensó la manera en que se espera que los estudiantes aprendan. El Proyecto utiliza el proceso de aprendizaje comprometido para proporcionar a los estudiantes las herramientas necesarias para adquirir el conocimiento experiencial. El objetivo es producir estudiantes que son seguros y autónomos en su aprendizaje y que tienen un alto sentido de relación con el contenido de lo que se enseña. Como resultado, la habilidad de los estudiantes para resolver problemas y su comprensión de la diversidad y del significado de la acción cívica han mejorado.

El Proyecto Horizonte intencionalmente proporciona oportunidades para que los estudiantes aprendan con personas y familias en los barrios y comunidades distintos a los suyos. Esta estrategia de exponer a los estudiantes a una variedad de grupos de la población ha demostrado ayudar a los estudiantes a entender mejor la salud y las desigualdades sociales y les ha permitido comenzar a entender las consecuencias de estas desigualdades. 
Cuando los estudiantes ingresan a entornos de aprendizaje servicios comunitarios lo hacen con un compañero estudiante $y$, siempre que sea posible, con un mentor que les presenta el entorno, el personal y a otras persona involucradas. Se pone mucho esfuerzo en la búsqueda de un entorno que ajuste bien con los estudiantes. Al mismo tiempo, es importante encontrar situaciones que pongan a prueba a los estudiantes, pero sin abrumarlos innecesariamente. Mientras se permite a veces que los estudiantes elijan sus parejas, son los profesores quienes tienen la última palabra. Dicho esto, la comunicación entre profesores y alumnos, y la valoración de las necesidades, deseos y preferencias individuales de los estudiantes son fundamentales para el éxito.

Una vez que los estudiantes son colocados en los entornos de aprendizaje servicio comunitarios del Proyecto Horizonte, su primera tarea es establecer una relación con los miembros de la comunidad, para así tratar de comprender las normas e idiosincrasia de la gente, y de reflexionar abiertamente sobre sus propios sentimientos, creencias y valores. Para lograr la humildad cultural se requiere que uno esté dispuesto a aprender de los demás de una manera abierta y sin prejuicios. Para las enfermeras practicantes que estudian en nuestro programa de Licenciatura en Ciencias en Enfermería esto a menudo significa que tienen que aprender una nueva forma de ser y comportarse con otra gente al asumir su papel profesional.

\section{Proyecto Horizonte - Fase 2: Desarrollo de consorcios y proyectos}

Uno de los primeros pasos en la creación de consorcios o asociaciones es estar abiertos y dispuestos a colaborar con los demás y a dedicar tiempo y energía para encontrar cosas en común. Los profesores del Proyecto Horizonte sirven de modelo demostrando estrategias para la buena comunicación con los demás, inclusive con los estudiantes y con el personal y en los locales comunitarios. A los estudiantes se les enseña a utilizar técnicas de reflexión desde los primeros cursos del programa de Licenciatura y se les exige que sigan aplicando las mismas técnicas en su experiencia de aprendizaje servicio crítico.

Mientras que muchos estudiantes experimentan aprensión antes de entrar en los sitios, pronto expanden su nivel de comodidad cuando se dan cuenta que son capaces de expresar sus sentimientos, emociones y preocupaciones con el profesorado y con los demás. La conciencia de sí mismo, la autocrítica, la apertura, la reflexión y, finalmente, la trascendencia son elementos de la experiencia que son críticos para el desarrollo de la humildad cultural (10). Estas dimensiones de la humildad cultural son estimuladas y reforzadas en el Proyecto Horizonte a través de tutorías individualizadas, presentando modelos de conducta, por medio de consultas activas con los participantes, discusiones en grupo entre los estudiantes y profesores, conferencias impartidas por profesores y el requisito de escribir un diario reflexivo.

La creación de consorcios y el desarrollo de proyectos con el personal y otras participantes en los sitios de aprendizaje servicio comunitarios es un proceso continuo que comienza temprano. Durante las primeras semanas, el profesorado y los estudiantes colaboran con los participantes para diseñar e implementar roles desafiantes y significativos para todos los participantes. La reciprocidad y la mutualidad son componentes necesarios de las asociaciones democráticas (16). En el Proyecto Horizonte esto se logra cuando los estudiantes asumen un papel activo en el establecimiento y mantenimiento de las conexiones, la construcción de confianza y la evaluación de las necesidades del entorno de aprendizaje servicio. Estas actividades ayudan a los estudiantes en su capacidad de llegar a tener un sentido de relación y sirven para fomentar la confianza, la competencia y autonomía. Tal y como lo validaron Levesque-Bristol et al. (14), estos componentes 
mejoran la percepción de la propia eficacia de la experiencia de aprendizaje servicio de los estudiantes.

Los estudiantes del Proyecto Horizonte aprenden a valorar las voces de los participantes de la comunidad y a diseñar, coordinar y ejecutar la totalidad de sus actividades en conjunto con ellos. Este tipo de decisiones aunadas requiere que los estudiantes abandonen actitudes paternalistas y dejen de lado la idea de que son los únicos poseedores de los conocimientos que sirven para determinar qué es lo mejor para los demás.

La transformación de la conciencia, las creencias y en última instancia el comportamiento resulta más fácil para algunos estudiantes que para otros. La tutoría continúa por el profesorado, la tutoría entre pares por sus propios compañeros y el apoyo y la orientación de los colaboradores comunitarios son métodos que utiliza el Proyecto Horizonte para ayudar a los estudiantes en el proceso de transformación hacia una práctica más democrática. Estos mecanismos para aumentar la comprensión y el respeto entre los alumnos y los grupos de población que atienden son esenciales para las iniciativas de aprendizaje servicio crítico. $\mathrm{Al}$ mismo tiempo, cuando se aplican correctamente, también sirven para mejorar la humildad cultural de los estudiantes y la de los profesores.

\section{Proyecto Horizonte - Fase 3: Implementa- ción, evaluación y sostenibilidad}

Una de las claves para producir un cambio significativo en una comunidad es el grado en que los participantes de la comunidad tienen el conocimiento y el poder para mediar y sostener el cambio. Iniciativas educativas solidarias llevadas a cabo por los alumnos sin la aportación completa y colaboración de los participantes de la comunidad pueden tener éxito temporal, pero frecuentemente, los cambios no pueden ser sostenidos una vez que los estudiantes salen del entorno. Los estudiantes aprenden que la creación de inicia- tivas significativas impulsadas directamente por miembros de la comunidad, esto es, iniciativas en que la comunidad se involucra en su diseño, implementación y evaluación tienen una probabilidad mucho mayor de ser sostenidas por más largo tiempo.

A los estudiantes del Proyecto Horizonte se les enseña el método de co-creación de todas sus iniciativas siempre junto con miembros de la comunidad en los locales de aprendizaje servicio y también la construcción de un mecanismo para que el cambio sea sostenido después de que ellos salgan del programa. Los estudiantes son responsables de evaluar el entorno de la comunidad, de la identificación de problemas junto con miembros de la comunidad y de la investigación de la literatura para proporcionar el conocimiento más actualizado a la comunidad. Los miembros de la comunidad ayudan en la investigación proporcionando orientación y clarificación a los estudiantes y sugiriendo estrategias de intervención que están en sintonía con los estilos de la comunidad. Los estudiantes crean decisiones racionales para un plan de acción, ejecución y evaluación junto con miembros de la comunidad. Los recursos disponibles y el capital humano son una parte de todos los planes, así como los mecanismos para la continuación del programa después de que los estudiantes salgan. Hay evidencia de que incluso los programas mas pequeños pueden hacer una gran diferencia en términos de mejora de la salud pública cuando se aplican correctamente.

Los estudiantes son asignados a entornos de aprendizaje servicio por un día a la semana durante todo un año académico - un total de dos semestres de 14 semanas. Esta práctica extendida da a los estudiantes tiempo suficiente para construir una buena relación y crear conexiones formidables con el personal del entorno y otras participantes Las iniciativas planificadas conjuntamente involucran a los estudiantes y a los miembros de la comunidad en la acción y ayudan 
a los estudiantes a aprender cómo piensan y actúan los miembros de la comunidad. Los estudiantes a menudo son sorprendidos por el nivel de ingenio, la habilidad y la iniciativa que exhiben los miembros de la comunidad. Frecuentemente, los estudiantes repiensan sus actitudes y prejuicios sobre los grupos marginados y se cuestionan los mitos, estereotipos y etiquetas que comúnmente se les asignan. Estos elementos trabajan juntos para fortalecer la naturaleza y la calidad de los consorcios y asociaciones.

Foster (11) plantea que "el fruto de una buena colaboración es la confianza que profundiza las relaciones con los otros". Las conexiones construidas en Proyecto Horizonte se renuevan y se solidifican cada año cuando los profesores regresan con nuevos estudiantes al mismo entorno comunitario para restablecer relaciones. El legado del proyecto en la comunidad y la confianza resultante de largo tiempo de interacción ayudan a inspirar y mantener la humildad cultural en las nuevas generaciones de estudiantes.

Los profesores del Proyecto Horizonte hacen un esfuerzo concertado para ayudar a los estudiantes y miembros de la comunidad a comprender los problemas estructurales globales a nivel macro y su impacto en las desigualdades sociales y de salud, así como las formas de invertir y mejorar dichas circunstancias a través de acciones orientado hacia la justicia social. Después de la construcción de relaciones y la colaboración en una serie de actividades con los miembros de la comunidad durante un período de meses, los estudiantes se abren más a las sugerencias que están basados en la justicia, y no a aquéllas basadas en el miedo. Al final de la experiencia con Proyecto Horizonte, el estudiante suele alcanzar todas las dimensiones de la humildad cultural (la autorreflexión, la auto crítica, la apertura y la trascendencia), quedando así preparado para asumir el papel de proveedor de servicios de salud bien informado y de agente de cambio con una perspectiva de orientación democrática.

\section{CONSIDERACIONES FINALES}

Las desigualdades estructurales y de salud plagan el tejido social de la sociedad de EEUU. Las clases sociales se mantienen separadas a través de la estratificación social, el miedo y la falta de comunicación. La educación crítica sobre la conexión entre la pobreza a nivel local y la economía política a nivel macro puede ayudar a explicar la desigualdad existente. Los profesionales sanitarios, incluyendo las enfermeras, pueden asumir un papel importante en la creación de colaboraciones, consorcios y asociaciones que fomenten el entendimiento y el respeto entre los proveedores de salud y sus clientes. La preparación de las nuevas generaciones de enfermeras y otros proveedores de atención médica con un sentido de responsabilidad y justicia social es una forma de revolucionar las profesiones dedicadas al cuidado de la salud. Hacer esto a principios de la carrera puede tener un efecto prolongado y dramático entre los proveedores de atención médica. El aprendizaje servicio crítico puede ser utilizado como una pedagogía crítica para ayudar a organizar los programas académicos y los consorcios entre las universidades y las comunidades.

El propósito de este artículo fue describir los principales aspectos del Proyecto Horizonte, un consorcio innovador con base universitaria dedicado a la salud y la promoción social que fomenta la humildad cultural utilizando la metodología de aprendizaje servicio crítico en colaboración conjunta con comunidades urbanas.

El Proyecto Horizonte es un programa de apoyo social y a la salud con base en la universidad que se asocia con grupos de gente en comunidades marginadas para aumentar su capacidad de salud y bienestar. El Proyecto proporciona un marco para mejorar la salud y el conocimiento social a través de la acción y el cambio basado en la evidencia. El Proyecto Horizonte utiliza el aprendizaje 
servicio crítico y la pedagogía crítica para construir y fortalecer la capacidad de humildad cultural y las asociaciones democráticas.

El aprendizaje servicio crítico y la pedagogía crítica pueden ayudar a aliviar las desigualdades de poder entre estudiantes universitarios y los miembros de la comunidad e inspirar la práctica democrática de los estudiantes y los proveedores de servicios de salud.

Este artículo describe los resultados de una exitosa y comprometida asociación de largo plazo y demuestra la eficacia de esta colaboración como instrumento para el desarrollo y la mejora de todas las dimensiones de la humildad cultural entre los profesionales de la salud. La replicación de un proyecto de promoción social y de salud, tal como el Proyecto Horizonte, puede intentarse en otros lugares para ver si son posibles resultados similares. Esfuerzos como éste pueden tener efectos dramáticos y positivos en los proveedores de servicios de salud, así como en los grupos de gente que vive en comunidades marginadas.

\section{REFERENCIAS}

1. U.S. Dept of Health and Human Services, Agency for Healthcare Research and Quality. 2010 National Healthcare Disparities Report. Rockville: AHRQ; 2011.

2. U.S. Department of Health and Human Services. Healthy People 2010: Understanding and improving health. $2 \mathrm{a}$ ed. Washington, DC: U.S. Government Printing Office; 2000.

3. Committee on Public Health Strategies to Improve Health; Board on Population Health and Public Health Practices; Institute of Medicine. For The Public's Health: Investing in a Healthier Future. Washington, DC: National Academies Press; 2012.

4. Smedley BD, Stith AY, Nelson AR. Une- qual Treatment: Confronting Racial and Ethnic Disparities in Healthcare. Washington, DC: National Academies Press; 2003.

5. Breda KL, Barbee EL, Zadoroznyj M. Nursing in the United States. En: Breda KL, Elling RH, eds. Nursing and globalization in the Americas: A critical perspective. Critical, Approaches in the Health Social Sciences Series. Amityville, NY: Baywood; 2009. p. 275-322.

6. Baer H, Singer M, Susser I. Medical Anthropology and the World System. 2a ed. Westport, CT: Praeger Publishers; 2003.

7. U.S. Dept of Health and Human Services, Agency for Healthcare Research and Quality. 2004 National Healthcare Disparities Report. Rockville: AHRQ; 2004.

8. University of Hartford. College of Education, Nursing and Health Professions: Center for Health, Care \& Well-Being [Internet]. West Hartford (CT): University of Hartford, Institute for Translational Research; 2012 [actualizado 2012; citado julio 2012]. Disponible en http:// www.hartford.edu/enhp/itr/chcwb/default.aspx

9. Miller S. Cultural Humility is the First Step to Becoming Global Care Providers. J Obstet Gynecol Neonatal Nurs. 2009; 38(1): 92-3.

10. Ortega RM, Faller KC. Training child welfare workers from an intersectional cultural humility perspective: a paradigm shift. Child Welfare. 2011; 90(5): 27-49.

11. Foster J. Cultural humility and the importance of long-term relationships in international partnerships. J Obstet Gynecol Neonatal Nurs. 2009; 38(1): 10007.

12. Cruess SR, Cruess RL, Steinert Y. Linking the teaching of professionalism to the social contract: a call for cultural humility. Med Teach. 2010; 32(5):357-59.

13. Bamber P, Hankin L. Transformative learning through service-learning: no 
passport required. Education + Training. 2011; 53(2/3): 190-206.

14. Levesque-Bristol C, Knapp D, Fisher B. The effectiveness of service-learning: It's not always what you think. Journal of Experiential Education. 2010; 33(3): 20824.

15. Davis DJ. Training transformative leaders through critical service-learning. Techni- ques [Internet]. Octubre 2007 [citado 12 julio 2012]; 82(7): 12-3. Disponible en: http://connection.ebscohost.com/c/articles/27077045/training-transformativeleaders-through-critical-service-learning

16. Breda KL, Wright MGM. Enhancing nursing knowledge through democratic cross-national collaboration. Texto context-enferm. 2011; 20(3): 392-98. 